\title{
ALGEBRAIC INVARIANT CURVES AND FIRST INTEGRALS FOR RICCATI POLYNOMIAL DIFFERENTIAL SYSTEMS
}

\author{
JAUME LLIBRE AND CLÀUDIA VALLS
}

(Communicated by Yingfei Yi)

\begin{abstract}
We study the algebraic invariant curves and first integrals for the Riccati polynomial differential systems of the form $x^{\prime}=1, y^{\prime}=a(x) y^{2}+$ $b(x) y+c(x)$, where $a(x), b(x)$ and $c(x)$ are polynomials. We characterize them when $c(x)=\kappa(b(x)-\kappa a(x))$ for some $\kappa \in \mathbb{C}$. We conjecture that algebraic invariant curves and first integrals for these Riccati polynomial differential systems only exist if $c(x)=\kappa(b(x)-\kappa a(x))$ for some $\kappa \in \mathbb{C}$.
\end{abstract}

\section{Introduction AND STATEMENT OF the MAIN RESUlts}

One of the more classical problems in the qualitative theory of planar differential equations depending on parameters is to characterize the existence or not of first integrals in the function of these parameters.

Let $x$ and $y$ be complex variables. We consider the system

$$
\dot{x}=1, \quad \dot{y}=a(x) y^{2}+b(x) y+c(x),
$$

where $a(x), b(x)$ and $c(x)$ are $C^{1}$ functions on $x$ and the prime denotes a derivative with respect to the time $t$ that can be either real or complex. In fact, if $a(x) c(x) \not \equiv 0$ these systems are called Riccati differential systems, if $a(x) \not \equiv 0$ and $c(x) \equiv 0$ they are a particular case of Bernouilli differential systems, and if $a(x) \equiv 0$, then they are linear differential systems.

Differential systems (1) are named after Count Jacobo Francesco Riccati (16761754). These equations have been intensively studied, and hundreds of applications have been found. Thus looking at MathSciNet there are more than 4000 papers having in their title the words "Riccati equations". These equations have been studied in many books; see for instance $7,9,13,15,19$. . They are important since they can be used to solve second-order ordinary differential equations. An important application of the Riccati differential systems is to the 3rd order Schwarzian differential equation which appears in the theory of conformal mapping and univalent functions; see [14] for more details and references.

Our interest is on the Riccati polynomial differential systems (1), i.e. when the functions $a(x), b(x)$ and $c(x)$ are polynomials. More precisely, we want to study

Received by the editors November 18, 2011 and, in revised form, December 13, 2011; May 1, 2012; September 6, 2012; and October 28, 2012.

2010 Mathematics Subject Classification. Primary 34C05, 34A34, 34C14.

Key words and phrases. Algebraic first integrals, algebraic invariant curves, Riccati polynomial differential equations.

The first author was partially supported by the MICINN/FEDER grant MTM2008-03437, AGAUR grant 2009SGR-410, ICREA Academia and FP7-PEOPLE-2012-IRSES-316338.

The second author was partially supported by the FCT through CAMGDS, Lisbon. 
the invariant algebraic curves of the Riccati polynomial differential systems and their algebraic integrability. But this problem has a long history. Thus when the functions $a(x), b(x)$ and $c(x)$ are rational, the irreducible invariant algebraic curves $f(x, y)=0$ ( $f$ is a polynomial) only can have as a polynomial in the variable $y$ degrees 1,2,4,6 and 12; see for more details Kovacic [10] (1986), who provided a constructive algorithm for finding invariant algebraic curves. In any case, before arriving at this nice result there were many preliminary studies. The first known algorithm for finding invariant algebraic curves was due to Liouville [11] (1833). Later on Fuchs [5] (1878) and Pépin [16] (1881) also provided algorithms for finding invariant algebraic curves; see Ulmer and Weil [18] (1996). Singer in [17] (1979) stated that all algorithms of these authors did not provide a complete description procedure and that the first complete algorithm was due to Baldassarri and Dwork [1] (1979).

The vector field associated to system (1) is

$$
X=\frac{\partial}{\partial x}+\left(a(x) y^{2}+b(x) y+c(x)\right) \frac{\partial}{\partial y} .
$$

The two main objectives of this paper are to study the algebraic first integrals and the invariant algebraic curves of the Ricatti polynomial differential systems. Partial results in this direction were obtained by Żołądek in [20, where the author characterized the possible algebraic solutions for the Riccati polynomial differential systems (11) with $a(x)=1$ and $b(x)=0$.

Let $U \subset \mathbb{C}^{2}$ be an open set. We say that the non-constant function $H: \mathbb{C}^{2} \rightarrow \mathbb{C}$ is a first integral of the polynomial vector field $X$ on $U$ if $H(x(t), y(t))$ is constant for all values of $t$ for which the solution $(x(t), y(t))$ of $X$ is defined on $U$. Clearly $H$ is a first integral of $X$ on $U$ if and only if $X H=0$ on $U$.

An algebraic first integral is a first integral which is an algebraic function. An algebraic function $f(x)=C$ is a solution of

$$
q_{0}(x)+q_{1}(x) C+q_{2}(x) C^{2}+\cdots+q_{s-1}(x) C^{s-1}+C^{s}=0,
$$

where $q_{j}(x)$ are rational functions of $x$ and $s$ is the smallest positive integer for which such a relation holds. Of course, all rational functions are algebraic functions.

Theorem 1. The Riccati polynomial differential system (11) with

$$
c(x)=\kappa(b(x)-\kappa a(x))
$$

for some $\kappa \in \mathbb{C}$ has an algebraic first integral $H=H(x, y)$ if and only if $b(x)=$ $2 \kappa a(x)$ (and in this case $c(x)=\kappa^{2} a(x)$ ), and the algebraic first integrals are algebraic functions in the variable $H=\int a(x) d x+1 /(y+\kappa)$.

For proving Theorem 1 we need to characterize the Darboux polynomials of the Ricatti polynomial differential systems. Let $h=h(x, y) \in \mathbb{C}[x, y] \backslash \mathbb{C}$. As usual $\mathbb{C}[x, y]$ denotes the ring of all complex polynomials in the variables $x$ and $y$. We say that $h=0$ is an invariant algebraic curve of the vector field $X$ associated to the Riccati polynomial differential system (11) if it satisfies

$$
\frac{\partial h}{\partial x}+\left(a(x) y^{2}+b(x) y+c(x)\right) \frac{\partial h}{\partial y}=K h,
$$


and the polynomial $K=K(x, y) \in \mathbb{C}[x, y]$ is called the cofactor of $h=0$ and has degree at most

$$
\max \{2+\operatorname{deg} a(x), 1+\operatorname{deg} b(x), \operatorname{deg} c(x)\}-1 .
$$

When $h=0$ is an invariant algebraic curve we also say that $h$ is a Darboux polynomial of the Riccati polynomial differential system. Note that a polynomial first integral is a Darboux polynomial with zero cofactor.

We conjecture that the unique invariant algebraic curves and algebraic first integrals of the Riccati polynomial differential systems exist when

$$
c(x)=\kappa(b(x)-\kappa a(x)) .
$$

Of course, if this conjecture holds, then Theorem 1 provides all the invariant algebraic curves and algebraic first integrals of the Riccati polynomial differential systems.

We say that $a(x)$ and $b(x)$ satisfy condition $P$ if

$$
\exp \left(-\int(b(x)-2 \kappa a(x)) d x\right) \int a(x) \exp \left(\int(b(s)-2 \kappa a(s)) d s\right) d x
$$

is a polynomial in the variable $x$. Note that if $b(x)=2 \kappa a(x)$, then $a(x)$ and $b(x)$ trivially satisfy condition $P$.

We must mention that all the indeterminant integrals that appear in this paper do not contain an integral constant.

We now show the following characterization for condition $P$ when $b(x) \neq 2 \kappa a(x)$.

Lemma 2. Assume that $b(x) \neq 2 \kappa a(x)$ and there exists a finite sequence $\left(Q_{j}\right)_{1 \leq j \leq N}$ of polynomials satisfying

$$
Q_{1}(x)=\frac{a(x)}{b(x)-2 \kappa a(x)}, \quad Q_{N}(x)=1,
$$

and for $j=1, \ldots, N-2$,

$$
Q_{j+1}(x)=\frac{Q_{j}^{\prime}(x)}{b(x)-2 \kappa a(x)} .
$$

Then $a(x)$ and $b(x)$ satisfy condition $P$ and

$$
\begin{aligned}
\exp ( & \left.-\int(b(x)-2 \kappa a(x)) d x\right) \int a(x) \exp \left(\int(b(s)-2 \kappa a(s)) d s\right) d x \\
& =Q_{1}(x)-Q_{2}(x)+\cdots+(-1)^{N} A_{N-1}(x)+(-1)^{N+1} .
\end{aligned}
$$

The proof of Lemma 2 is given in Section 2

Theorem 3. The following statements hold for the Riccati polynomial differential systems (11):

(a) They have no polynomial first integrals.

(b) When $c(x)=\kappa(b(x)-\kappa a(x))$ for some $\kappa \in \mathbb{C}$, they have the irreducible Darboux polynomial $f=y+\kappa$ with the non-zero cofactor $K=b(x)+$ $a(x)(y-\kappa)$. If additionally, $a(x)$ and $b(x)$ satisfy condition $P$, then

$f=1+(y+\kappa) \exp \left(-\int(b(x)-2 \kappa a(x)) d x\right) \int a(x) \exp \left(\int(b(s)-2 \kappa a(s)) d s\right) d x$ is another irreducible Darboux polynomial with the non-zero cofactor $K=$ $a(x)(y+\kappa)$. 
The proofs of Theorems 1 and 3 are given in Section 3 .

Let $\mathbb{C}[x, y]$ be the ring of all complex polynomials in the variables $x$ and $y$ respectively. We note, from Theorem 3, that all irreducible Darboux polynomials in $\mathbb{C}[x, y]$ for the Riccati polynomial differential systems with $c(x)=\kappa(b(x)-$ $\kappa a(x))$ have degree 1 in the variable $y$. So, there is a big difference between the Riccati rational differential systems and the Riccati polynomial differential systems, because as we have mentioned for the rational ones their invariant algebraic curves can have degrees $1,2,4,6$ and 12 in the variable $y$.

Remark 4. It is known that if we have a particular solution of a Riccati differential equation

$$
\frac{d y}{d x}=a(x) y^{2}+b(x) y+c(x),
$$

then this can be reduced to a Bernouilli differential equation which is always integrable. Note that under the assumption $c(x)=\kappa(b(x)-\kappa a(x))$ Theorem 1 characterizes the existence of an algebraic first integral without using any particular solution.

\section{Proof of Lemma 2}

In this section we prove Lemma 2. Assume that $b(x) \neq 2 \kappa a(x)$ and that there exists a finite sequence $\left(Q_{j}\right)_{1 \leq j \leq N}$ of polynomials satisfying

$$
Q_{1}(x)=\frac{a(x)}{b(x)-2 \kappa a(x)}, \quad Q_{N}(x)=1,
$$

and for $j=1, \ldots, N-2$,

$$
Q_{j+1}(x)=\frac{Q_{j}^{\prime}(x)}{b(x)-2 \kappa a(x)} .
$$

Then, using the formula of integration by parts,

$$
\begin{aligned}
\int a(x) \exp \left(\int(b(s)-2 \kappa a(s)) d s\right) d x \\
=\int Q_{1}(x)(b(x)-2 \kappa a(x)) \exp \left(\int(b(s)-2 \kappa a(s)) d s\right) d x \\
=Q_{1}(x) \exp \left(\int(b(x)-2 a(x) \kappa) d x\right)-\int Q_{1}^{\prime}(x) \exp \left(\int(b(s)-2 \kappa a(s)) d s\right) d x \\
=Q_{1}(x) \exp \left(\int(b(x)-2 \kappa a(x)) d x\right) \\
\quad-\int Q_{2}(x)(b(x)-2 \kappa a(x)) \exp \left(\int(b(s)-2 \kappa a(s)) d s\right) d x \\
=\left(Q_{1}(x)-Q_{2}(x)\right) \exp \left(\int(b(x)-2 \kappa a(x)) d x\right) \\
\quad+\int Q_{2}^{\prime}(x) \exp \left(\int(b(s)-2 \kappa a(s)) d s\right) d x \\
=\cdots
\end{aligned}
$$




$$
\begin{aligned}
= & \left(Q_{1}(x)-Q_{2}(x)+\cdots+(-1)^{N} Q_{N-1}(x)\right) \exp \left(\int(b(x)-2 \kappa a(x)) d x\right) \\
& +(-1)^{N+1} \int Q_{N-1}^{\prime}(x) \exp \left(\int(b(s)-2 \kappa a(s)) d s\right) d x \\
= & \left(Q_{1}(x)-Q_{2}(x)+\cdots+(-1)^{N} Q_{N-1}(x)\right) \exp \left(\int(b(x)-2 \kappa a(x)) d x\right) \\
& +(-1)^{N+1}(b(x)-2 \kappa a(x)) \int \exp \left(\int(b(s)-2 \kappa a(s)) d s\right) d x \\
= & \left(Q_{1}(x)-Q_{2}(x)+\cdots+(-1)^{N} Q_{N-1}(x)+(-1)^{N+1}\right) \\
& \times \exp \left(\int(b(x)-2 \kappa a(x)) d x\right) .
\end{aligned}
$$

Now it readily follows from the above identity that

$$
\begin{aligned}
& \exp \left(-\int(b(x)-2 \kappa a(x)) d x\right) \int a(x) \exp \left(\int(b(s)-2 \kappa a(s)) d s\right) d x \\
& =Q_{1}(x)-Q_{2}(x)+\cdots+(-1)^{N} Q_{N-1}(x)+(-1)^{N+1} .
\end{aligned}
$$

This completes the proof of the lemma.

\section{Proofs of Theorems 1$]$ and 3}

We separate the proof of Theorem 3 in several steps.

Lemma 5. Riccati polynomial differential systems (1) have no polynomial first integrals.

Proof. We proceed by contradiction. Let $H$ be a polynomial first integral of system (11), that is,

$$
\frac{\partial H}{\partial x}+\left(a(x) y^{2}+b(x) y+c(x)\right) \frac{\partial H}{\partial y}=0 .
$$

We write $H$ as a polynomial in the variable $y$, i.e.

$$
H(x, y)=\sum_{j=0}^{m} h_{j}(x) y^{j}, \quad \text { where } h_{j}(x) \text { is a polynomial in the variable } x .
$$

Without loss of generality we can assume that $h_{m}(x) \neq 0$.

Computing the coefficient of degree $m+1$ in the variable $y$ in (2) we get that

$$
m a(x) h_{m}(x)=0 .
$$

Since $a(x) h_{m}(x) \neq 0$, we must have $m=0$. Then $H=h_{0}(x)$. In view of (2) we get that $H$ satisfies

$$
H^{\prime}(x)=0, \quad \text { that is } H(x) \in \mathbb{C},
$$

a contradiction with the fact that $H$ is a polynomial first integral.

The proof of the following proposition is well-known and can be found in [2]

Proposition 6. We suppose that $h \in \mathbb{C}[x, y]$ and let $h=h_{1}^{n_{1}} \cdots h_{r}^{n_{r}}$ be its factorization in irreducible factors over $\mathbb{C}[x, y]$. Then for a polynomial system (1) $h=0$ is an invariant algebraic curve with cofactor $K_{h}$ if and only if $h_{i}=0$ is an invariant algebraic curve for each $i=1, \ldots, r$ with cofactor $K_{h_{i}}$. Moreover, $K=n_{1} K_{h_{1}}+\cdots+n_{r} K_{h_{r}}$. 
In view of Proposition [6 to study the Darboux polynomials with a non-zero cofactor it is enough to study the irreducible ones.

Lemma 7. Let $h=h(x, y)$ be an irreducible Darboux polynomial of the Riccati polynomial differential system (11) with cofactor $K \neq 0$. Then $K=K_{0}(x)+m a(x) y$ with $m$ a non-negative integer.

Proof. We define the degree $n$ of the Riccati polynomial differential system (11) as

$$
n=\max \{2+\operatorname{deg} a(x), 1+\operatorname{deg} b(x), \operatorname{deg} c(x)\} .
$$

We note that $n \geq 2$. Then the cofactor $K$ has degree at most $n-1$. We write it as $K(x, y)=\sum_{j=0}^{n-1} K_{j}(x) y^{j}$, where $K_{j}=K_{j}(x)$ is a polynomial in the variable $x$ and has at most degree $n-1-j$. Since $h$ is a Darboux polynomial of system (1) with cofactor $K$ it satisfies

$$
\frac{\partial h}{\partial x}+\left(a(x) y^{2}+b(x) y+c(x)\right) \frac{\partial h}{\partial y}=\left(\sum_{j=0}^{n-1} K_{j}(x) y^{j}\right) h .
$$

We write $h$ as a polynomial in the variable $y$, i.e. $h(x, y)=\sum_{j=0}^{m} h_{j}(x) y^{j}$, where each $h_{j}(x)$ is a polynomial in the variable $x$. Without loss of generality we can assume that $h_{m}(x) \neq 0$.

Assume $n \geq 3$. Computing the coefficient of $y^{n+m-1}$ in (3) we get

$$
0=K_{n-1}(x) h_{m}(x), \quad \text { that is, } \quad K_{n-1}(x)=0 .
$$

Now proceeding inductively, we can show that for $\ell=n-1, n-2, \ldots, 3$, we have $K_{\ell}(x)=0$. Therefore, $K=K_{0}(x)+y K_{1}(x)$. So, for $n \geq 2$ we have that always the cofactor of an irreducible Darboux polynomial is of the form $K=K_{0}(x)+y K_{1}(x)$.

Now computing the coefficient of $y^{m+1}$ in (3) we get

$$
\operatorname{ma}(x) h_{m}(x)=h_{m}(x) K_{1}(x),
$$

that is, $\left(m a(x)-K_{1}(x)\right) h_{m}(x)=0$. Using the fact that $h_{m}(x) \neq 0$ we must have $K_{1}(x)=m a(x)$ with $m$ a non-negative integer. This completes the proof.

Proposition 8. The Riccati polynomial differential systems (1) with

$$
c(x)=\kappa(b(x)-\kappa a(x))
$$

for some $\kappa \in \mathbb{C}$ have the irreducible Darboux polynomial $f=y+\kappa$ with the non-zero cofactor $K=b(x)+a(x)(y-\kappa)$. If additionally, $a(x)$ and $b(x)$ satisfy condition $P$, then

$f=1+(y+\kappa) \exp \left(-\int(b(x)-2 \kappa a(x)) d x\right) \int a(x) \exp \left(\int(b(s)-2 \kappa a(s)) d s\right) d x$

is another irreducible Darboux polynomial with the non-zero cofactor

$$
K=a(x)(y+\kappa) .
$$

Proof. We write the Riccati polynomial differential system (1) as the differential equation

$$
\frac{d y}{d x}=a(x) y^{2}+b(x) y+c(x), \quad y=y(x)
$$


Then, using Lemma 7 the Darboux polynomial $h=h(x, y)=h(x, y(x))$ satisfies

$$
\frac{d h}{d x}=\frac{\partial h}{\partial x}+\frac{\partial h}{\partial y}\left(a(x) y^{2}+b(x) y+c(x)\right)=\left(K_{0}(x)+m a(x) y\right) h, \quad m \in \mathbb{N},
$$

or equivalently,

$$
\log h=K+\int\left(K_{0}(x)+m a(x) y\right) d x, \quad \text { where } \quad K \in \mathbb{C} .
$$

Hence

$$
h=h(x, y(x))=C \exp \left(\int\left(K_{0}(x)+m a(x) y\right) d x\right), \quad C \in \mathbb{C} \backslash\{0\} .
$$

Now we write

$$
a(x) y^{2}+b(x) y+c(x)=\left(y+\Gamma_{1}(x)\right)\left(a(x) y+\Gamma_{2}(x)\right)
$$

where

$$
\Gamma_{1}(x)=\frac{b(x)}{2 a(x)}-\frac{\sqrt{b(x)^{2}-4 a(x) c(x)}}{2 a(x)}, \quad \Gamma_{2}(x)=\frac{b(x)}{2}+\frac{\sqrt{b(x)^{2}-4 a(x) c(x)}}{2} .
$$

Since $c(x)=\kappa(b(x)-\kappa a(x))$ for some $\kappa \in \mathbb{C}$ we have that $\Gamma_{1}(x)=\kappa \in \mathbb{C}$ and $\Gamma_{2}(x)=b(x)-\kappa a(x)$. Hence, it follows from (4) and (7) that

$\frac{d y}{d x}=(y+\kappa)\left(a(x) y+\Gamma_{2}(x)\right), \quad$ that is, $\quad a(x) y+\Gamma_{2}(x)=\frac{d y / d x}{y+\kappa}=\frac{d \log (y+\kappa)}{d x}$,

which implies that (6) becomes

$$
\begin{aligned}
h & =C \exp \left(\int\left(K_{0}(x)-m \Gamma_{2}(x)+m\left(a(x) y+\Gamma_{2}(x)\right)\right) d x\right) \\
& =C \exp \left(\int\left(K_{0}(x)-m \Gamma_{2}(x)+m \frac{d \log (y+\kappa)}{d x}\right) d x\right) \\
& =C(y+\kappa)^{m} \exp \left(\int\left(K_{0}(x)-m(b(x)-\kappa a(x))\right) d x\right)
\end{aligned}
$$

with $C \in \mathbb{C} \backslash\{0\}$. Since $h$ must be a polynomial and $K_{0}(x)$ is a polynomial, we must have that the polynomial $K_{0}(x)=m(b(x)-\kappa a(x))$, and consequently $h(x)=C(y+\kappa)^{m}$. Now using the fact that $h$ is irreducible we get that $m=1$ and then $f=y+\kappa$ and $K=K_{0}(x)+m a(x) y=b(x)+a(x)(y-\kappa)$. Note that in this case the Ricatti polynomial differential systems become

$$
\dot{x}=1, \quad \dot{y}=(y+\kappa)(a(x)(y-\kappa)+b(x)) .
$$

Assume that $h=h(x, y)$ is another irreducible Darboux polynomial of system (8). Then if we denote by $\bar{h}=\bar{h}(x)$ the restriction of $h$ to $y=-\kappa$, we get from (5) that $\bar{h}$ satisfies

$$
\frac{d \bar{h}}{d x}=\left(K_{0}(x)-m \kappa a(x)\right) \bar{h} .
$$

Solving this linear differential equation we obtain

$$
\bar{h}=C e^{\int\left(K_{0}(x)-m \kappa a(x)\right) d x}, \quad C \in \mathbb{C} .
$$

It is clear that $\bar{h} \neq 0$; otherwise $h$ would be reducible. Hence, since $\bar{h}$ must be a polynomial we get $K_{0}(x)=m \kappa a(x)$. Then the cofactor of $h$ must be $K=$ $m a(x)(y+\kappa)$ and $h=C+(y+\kappa) g$ for some $C \in \mathbb{C}$ and $g \in \mathbb{C}[x, y]$. Since $h$ must be irreducible we have that $C \neq 0$. 
Now we consider two cases.

Case 1: $a(x)$ and $b(x)$ do not satisfy condition $P$. Clearly $g=g(x, y)$ satisfies

$$
\frac{\partial g}{\partial x}+(a(x) y+b(x)-\kappa a(x))\left(g+(y+\kappa) \frac{\partial g}{\partial y}\right)=m a(x)(C+(y+\kappa) g),
$$

where we have simplified the common factor $y+\kappa$. Now let $\bar{g}=\bar{g}(x)$ be the restriction of $g$ to $y=-\kappa$. We get that $\bar{g}$ satisfies

$$
\frac{d \bar{g}}{d x}+(b(x)-2 \kappa a(x)) \bar{g}=m C a(x) .
$$

Solving this linear differential equation we get

$$
\bar{g}=e^{\int(-b(x)+2 \kappa a(x)) d x}\left(C_{1}+m C \int a(x) e^{\int(b(x)-2 \kappa a(x)) d x} d x\right)
$$

for some $C_{1} \in \mathbb{C}$, or equivalently,

$$
C_{1}=-m C \int a(x) e^{\int(b(x)-2 \kappa a(x)) d x} d x+\bar{g} e^{-\int(-b(x)+2 \kappa a(x)) d x},
$$

where $\bar{g}$ is a polynomial. Since $b(x) \neq 2 \kappa a(x)$, and the integrals $\bar{g} e^{-\int(-b(x)+2 \kappa a(x))} d x$ and $\int a(x) e^{\int(b(x)-2 \kappa a(x)) d x}$ are not constant, but $C_{1}$ is a constant, it follows that $C_{1}=0$, and

$$
m C \int a(x) e^{\int(b(x)-2 \kappa a(x)) d x}=\bar{g} e^{-\int(-b(x)+2 \kappa a(x)) d x} .
$$

But by assumption we know that

$$
e^{\int(-b(x)+2 \kappa a(x)) d x} \int a(x) e^{\int(b(x)-2 \kappa a(x)) d x} d x
$$

is not a polynomial, a contradiction with the fact that

$$
e^{\int(-b(x)+2 \kappa a(x)) d x} \int a(x) e^{\int(b(x)-2 \kappa a(x)) d x} d x=\bar{g} .
$$

Hence Case 1 does not hold.

Case 2: $a(x)$ and $b(x)$ do not satisfy condition $P$. In this case we have that

$f=1+(y+\kappa) \exp \left(-\int(b(x)-2 \kappa a(x)) d x\right) \int a(x) \exp \left(\int(b(s)-2 \kappa a(s)) d s\right) d x$

is an irreducible Darboux polynomial of the Riccati polynomial differential system (see (8)) with cofactor $K=a(x)(y+\kappa)$. Now we shall prove that it is the only one. We proceed by contradiction. Assume $h=h(x, y)$ is another irreducible Darboux polynomial of system (8). If we denote by $\tilde{h}=\tilde{h}(x)$ the restriction of $h$ to

$$
y=-\kappa-\exp \left(\int(b(x)-2 \kappa a(x)) d x\right) /\left(\int a(x) \exp \left(\int(b(s)-2 \kappa a(s)) d s\right) d x\right),
$$

from (5) the cofactor of $\tilde{h}$ is

$$
K=-m a(x) \exp \left(\int(b(x)-2 \kappa a(x)) d x\right) /\left(\int a(x) \exp \left(\int(b(s)-2 \kappa a(s)) d s\right)\right) .
$$

So, we have

$$
\frac{d \tilde{h}}{d x}=-\frac{m a(x) \exp \left(\int(b(x)-2 \kappa a(x)) d x\right)}{\int a(x) \exp \left(\int(b(s)-2 \kappa a(s)) d s\right) d x} \tilde{h} .
$$


Solving this linear differential equation we obtain

$$
\tilde{h}=C \exp \left(\int-\frac{m a(x) \exp \left(\int(b(x)-2 \kappa a(x)) d x\right)}{\int a(x) \exp \left(\int(b(s)-2 \kappa a(s)) d s\right) d x} d x\right), \quad C \in \mathbb{C} .
$$

It is clear that $\tilde{h} \neq 0$; otherwise $h$ would be reducible. Set

$$
f(x)=a(x) \exp \left(\int(b(x)-2 \kappa a(x)) d x\right), \quad F(x)=\int f(x) d x
$$

and

$$
P(x)=\exp \left(-\int(b(x)-2 \kappa a(x)) d x\right) \int a(x) \exp \left(\int(b(s)-2 \kappa a(s)) d s\right) d x .
$$

Then it follows from (10) that

$$
\begin{aligned}
\tilde{h} & =C \exp \left(\int-\frac{m f(x)}{F(x)} d x\right)=C \exp \left(\int-m \frac{d F(x)}{F(x)}\right)=C F^{-m} \\
& =C\left(\frac{\exp \left(-\int(b(x)-2 \kappa a(x)) d x\right)}{P(x)}\right)^{m} .
\end{aligned}
$$

Since $P(x)$ is a polynomial due to the fact that $a(x)$ and $b(x)$ satisfy condition $P$, whereas $\exp \left(-\int(b(x)-2 \kappa a(x)) d x\right)$ is a constant or an exponential function, we conclude that $\tilde{h}$ cannot be a polynomial. This contradiction shows that the system cannot have another irreducible Darboux polynomial. Hence, the proof of the proposition is completed.

Proof of Theorem 3. The proof of Theorem 3 is an immediate consequence of Lemma 5 and Proposition 8 .

To prove Theorem 1 we need the following two auxiliary results. For a proof of the first result see [12, and for a proof of the second one see 6 .

Proposition 9. The existence of a rational first integral for a polynomial differential system (11) implies either the existence of a polynomial first integral or the existence of two Darboux polynomials having the same non-zero cofactor.

Proposition 10. If a polynomial differential system has an algebraic first integral, then it has a rational first integral.

Proof of Theorem 1. From Proposition 10 to study the algebraic first integrals of the Riccati polynomial differential system (1) it is enough to study the rational ones. From Theorem 3 and Proposition 9, system (11) has a rational first integral if and only if it has two Darboux polynomials with the same non-zero cofactor. In view of Theorem 3. since $c(x)=\kappa(b(x)-\kappa a(x))$ for some $\kappa \in \mathbb{C}$, this is only possible if and only if $a(x)$ and $b(x)$ satisfy condition $P$. So, now we assume that $a(x)$ and $b(x)$ satisfy condition $P$ and we consider two different cases.

Case 1: $b(x) \neq 2 \kappa a(x)$. In this case the Darboux polynomials are of the form

$$
\begin{aligned}
&(y+\kappa)^{l}\left(1+(y+\kappa) \exp \left(-\int(b(x)-2 a(x) \kappa) d x\right)\right. \int a(x) \\
&\left.\times \exp \left(\int(b(s)-2 \kappa a(s)) d s\right) d x\right)^{m}
\end{aligned}
$$


with non-negative integers $l$ and $m$. By Proposition 6, the cofactor is

$$
l(b(x)+a(x)(y-\kappa))+m a(x)(y+\kappa) .
$$

Then, using the fact that $a(x) \neq 0$, in order to have a rational first integral we must have

(11) $l_{1}(b(x)+a(x)(y-\kappa))+m_{1} a(x)(y+\kappa)=l_{2}(b(x)+a(x)(y-\kappa))+m_{2} a(x)(y+\kappa)$,

with $l_{1}, l_{2}, m_{1}, m_{2}$ non-negative integers, $\left(l_{1}^{2}+m_{1}^{2}\right)\left(l_{2}^{2}+m_{2}^{2}\right) \neq 0$ and $\left(l_{1}, m_{1}\right) \neq$ $\left(l_{2}, m_{2}\right)$.

Computing the coefficient of $y$ in (11) we get that

$$
l_{1}+m_{1}-l_{2}-m_{2}=0, \quad \text { that is, } \quad l_{1}=l_{2}+m_{2}-m_{1} .
$$

Introducing it in (11), we get

$$
\left(m_{2}-m_{1}\right)(b(x)-2 \kappa a(x))=0,
$$

which is impossible because by assumptions $b(x) \neq 2 \kappa a(x)$, and $m_{2} \neq m_{1}$ otherwise, $\left(l_{1}, m_{1}\right)=\left(l_{2}, m_{2}\right)$, which is not possible. Hence in this case there are no algebraic first integrals.

Case 2: $b(x)=2 \kappa a(x)$. In this case $c(x)=\kappa^{2} a(x)$, and thus system (1) becomes

$$
x^{\prime}=1, \quad y^{\prime}=a(x)\left(y^{2}+2 \kappa y+\kappa^{2}\right)=a(x)(y+\kappa)^{2},
$$

whose rational first integral $H$ is

$$
H=\int a(x) d x+\frac{1}{y+\kappa}=\frac{1+(y+\kappa) \int a(x) d x}{y+\kappa} .
$$

This completes the proof of the theorem.

\section{ACKNOWLEDGEMENTS}

The authors thank the referee for comments and suggestions that helped us to improve this paper, mainly in the last step of the proof of Proposition 8 .

\section{REFERENCES}

[1] F. Baldassarri and B. Dwork, On second order linear differential equations with algebraic solutions, Amer. J. Math. 101 (1979), no. 1, 42-76, DOI 10.2307/2373938. MR527825(81d:34002)

[2] Colin Christopher and Jaume Llibre, Integrability via invariant algebraic curves for planar polynomial differential systems, Ann. Differential Equations 16 (2000), no. 1, 5-19. MR:1768817 (2001g:34001)

[3] Colin Christopher, Liouvillian first integrals of second order polynomial differential equations, Electron. J. Differential Equations (1999), No. 49, 7 pp. (electronic). MR.1729833 (2000i:34017)

[4] Colin Christopher, Jaume Llibre, and Jorge Vitório Pereira, Multiplicity of invariant algebraic curves in polynomial vector fields, Pacific J. Math. 229 (2007), no. 1, 63-117, DOI 10.2140/pjm.2007.229.63. MR2276503 (2008f:34065)

[5] L. Fuchs, Über die linearen Differentialgleichungen zweiter Ordnung welche algebraische Integrale besitzen, zweiter Abhandlung, J. für Math. 85 (1878).

[6] Alain Goriely, Integrability, partial integrability, and nonintegrability for systems of ordinary differential equations, J. Math. Phys. 37 (1996), no. 4, 1871-1893, DOI 10.1063/1.531484. MR 1380879 (97b:70021)

[7] Einar Hille, Ordinary differential equations in the complex domain, Pure and Applied Mathematics, Wiley-Interscience [John Wiley \& Sons], New York, 1976. MR.0499382(58 \#17266)

[8] E. L. Ince, Ordinary Differential Equations, Dover Publications, New York, 1944. MR.0010757 $(6,65 f)$ 
[9] E. Kamke, Differentialgleichungen "losungsmethoden und losungen", Col. Mathematik und ihre anwendungen vol. 18, Akademische Verlagsgesellschaft Becker und Erler Kom-Ges., Leipzig, 1943.

[10] Jerald J. Kovacic, An algorithm for solving second order linear homogeneous differential equations, J. Symbolic Comput. 2 (1986), no. 1, 3-43, DOI 10.1016/S0747-7171(86)80010-4. MR.839134 (88c:12011)

[11] J. Liouville, Sur la détermination des intégrales dont la valeur est algébrique, J. de l'École Polytechnique 22 (1833).

[12] Jaume Llibre and Clàudia Valls, Integrability of the Bianchi IX system, J. Math. Phys. 46 (2005), no. 7, 072901, 13, DOI 10.1063/1.1955453. MR2153554 (2006f:37087)

[13] George M. Murphy, Ordinary differential equations and their solutions, D. Van Nostrand Co., Inc., Princeton, N.J.-Toronto-London-New York, 1960. MR.0114953(22 \#5762)

[14] Zeev Nehari, Conformal mapping, Dover Publications Inc., New York, 1975. Reprinting of the 1952 edition. MR0377031 (51 \#13206)

[15] Andrei D. Polyanin and Valentin F. Zaitsev, Handbook of exact solutions for ordinary differential equations, 2nd ed., Chapman \& Hall/CRC, Boca Raton, FL, 2003. MR2001201 (2004g:34001)

[16] P.Th. Pépin, Méthode pour obtenir les intégrales algébriques des équations différentielles linéaires du second ordre, Atti. dell' Accad. Pont. de Nuovi Lincei XXXIV (1881), 243-388.

[17] Michael F. Singer, Liouvillian solutions of nth order homogeneous linear differential equations, Amer. J. Math. 103 (1981), no. 4, 661-682, DOI 10.2307/2374045. MR623132 (82i:12028)

[18] Felix Ulmer and Jacques-Arthur Weil, Note on Kovacic's algorithm, J. Symbolic Comput. 22 (1996), no. 2, 179-200, DOI 10.1006/jsco.1996.0047. MR.1422145 (97j:12006)

[19] William T. Reid, Riccati differential equations, Mathematics in Science and Engineering, vol. 86, Academic Press, New York, 1972. MR0357936 (50 \#10401)

[20] Henryk Żołądek, Polynomial Riccati equations with algebraic solutions, Differential Galois theory (Będlewo, 2001), Banach Center Publ., vol. 58, Polish Acad. Sci., Warsaw, 2002, pp. 219-231, DOI 10.4064/bc58-0-17. MR.1972457 (2004j:34200)

Departament de Matemàtiques, Universitat Autònoma de Barcelona, 08193 BellaTERra, BArcelona, CAtalonia, Spain

E-mail address: jllibre@mat.uab.cat

Departamento de Matemática, Instituto Superior Técnico, Universidade Técnica de Lisboa, Av. Rovisco Pais 1049-001, Lisboa, Portugal

E-mail address: cvalls@math.ist.utl.pt 